\title{
Mapping of a Lattice-Plane Tilting in a GaN Wafer Using Energy-Resolved X-Ray Diffraction Topography
}

\author{
Jaemyung Kim, ${ }^{1,2,3}$ Okkyun Seo, ${ }^{1,2,3}$ Chulho Song, ${ }^{3, \dagger}$ Satoshi Hiroi, ${ }^{2}$ Yanna Chen, ${ }^{2,3}$ Yoshihiro \\ Irokawa, ${ }^{1}$ Toshihide Nabatame, ${ }^{1}$ Yasuo Koide, ${ }^{1}$ and Osami Sakata ${ }^{1,2,3,}{ }^{*}$ \\ ${ }^{1}$ Center for GaN Characterization, Research Network and Facility Services Division, National Institute for \\ Materials Science (NIMS), Sengen, Tsukuba, Ibaraki 305-0047, Japan \\ ${ }^{2}$ Synchrotron X-ray Group, Research Center for Advanced Measurement and Characterization, NIMS, Kouto, \\ Sayo, Hyogo 679-5148, Japan \\ ${ }^{3}$ Synchrotron X-ray Station at Spring-8, Research Network and Facility Services Division, NIMS, Kouto, Sayo, \\ Hyogo 679-5148, Japan
}

(Received 3 September 2018; revised manuscript received 21 November 2018; published 28 February 2019)

\begin{abstract}
We investigate the local structures of a GaN substrate through an energy-resolved white $\mathrm{x}$-ray diffraction topography method. A section topography geometry is implemented at various sample positions for the purpose of wafer mapping. The obtained images at each position are piled up for the three-dimensional matrix and then sliced at a same energy position. The sliced images of the lower and higher diffraction energy show periodic bottomless and topless features originated from the local lattice tilting, respectively. It is revealed that the local lattice planes are tilted by $\pm 0.03^{\circ}$ around points in the periodic structures. This tilting angle is consistent with results obtained from monochromatic x-ray diffraction topography measurements. X-ray rocking curves obtained by a single-crystal $\mathrm{x}$-ray diffraction shows the tilting of local structure that is also consistent with the energy-resolved x-ray diffraction topography results.
\end{abstract}

DOI: 10.1103/PhysRevApplied.11.024072

\section{INTRODUCTION}

$\mathrm{GaN}$ is a promising material in the semiconductor industry, and its feasibility for use in optoelectronic devices and power devices has been demonstrated [1-3]. Both the manufacturing process and crystal growth technique have undergone drastic improvements since the development of GaN-based blue light-emitting diodes. However, only a few methods in estimating the crystal quality of GaN substrate have been developed, such as the $\mathrm{x}$-ray rocking curve method [4-6]. This rocking curve can be applied to several sample positions in outlining the crystallinity over the sample [6,7]. Another existing conventional way to evaluate the details of the wafer crystallinity is to employ monochromatic x-ray diffraction topography. Mahadik et al. has observed the defects on a GaN surface using a high-resolution x-ray diffraction topography

\footnotetext{
*sakata.osami@nims.go.jp

†Present address: NISSAN ARC, LTD., 1 Natsushima-cho, Yokosuka, Kanagawa 237-0061, Japan.

Published by the American Physical Society under the terms of the Creative Commons Attribution 4.0 International license. Further distribution of this work must maintain attribution to the author(s) and the published article's title, journal citation, and DOI.
}

technique that is combined with photoluminescence imaging [8]. In the case of orientation mapping based on the rocking curve imaging [9-15], it has been shown to have applications in the mapping of strain distributions, lattice plane tilting angles, and lattice constant deviations in the wafer. Another way to estimate the crystal quality is to use white x-ray diffraction topography [16-21]. This method enables us to get an absorption contrast image and Laue spots that contain orientation information. However, the contribution of the absorption contrast is composed of many wavelength components that need to consider the beam hardening effect (narrowing of the $\mathrm{x}$ ray band width) [22]. Even the diffracted Laue spots are polychromatic if there exist strains or misorientations. In order to avoid this trouble, a small-sized beam is required. Recently, Laue diffraction topography including the wire scanning method has been demonstrated for the purpose of obtaining three-dimensional lattice information with small-sized $\mathrm{x}$ rays $[16,17]$. Although this method is applicable to single-crystal as well as polycrystalline materials, it is hard to obtain information on a larger area. Accordingly, a method that enables the energy-resolved wafer scale imaging technique through white $\mathrm{x}$ rays is becoming more in demand.

In this paper, we suggest a method for observing the lattice-plane orientation and tilting of large-area $\mathrm{GaN}$ using 
an energy-resolved x-ray Laue diffraction method. Our findings indicate that the lattice-plane orientation around the periodic structure is inclined approximately $\pm 0.03^{\circ}$ relative to the higher crystallinity area.

\section{EXPERIMENTAL DETAILS}

The experiment is performed at BL28B2, SPring-8, Japan. The samples used in this study consist of an undoped $1-\mu \mathrm{m}$-thick $\mathrm{GaN}$ layer and a $2-\mu \mathrm{m}$-thick $\mathrm{Mg}$ doped layer on $\mathrm{GaN}$ (0001) substrates that have periodic structures in the in-plane directions. A thick GaN substrate is grown on the periodic array of GaN seed crystals in order to minimize the strain and dislocation density with the hydride vapor-phase epitaxy (HVPE) method. The substrate thickness is about $300 \mu \mathrm{m}$ such that the diffracted $\mathrm{x}$ rays are mainly originated from the bulk substrate rather than epitaxial layers. The sample size is $8 \times 8 \mathrm{~mm}^{2}$. To determine the exact orientation of the $\mathrm{GaN}$ substrate, we change the beam size to $0.1 \times 0.1 \mathrm{~mm}^{2}$. The observed Laue spot is compared with the calculation using the software LAUEPT [23]. The lattice constants $a$ and $c$ are 3.186 and $5.189 \AA$, respectively. The incident angle is adjusted to $3^{\circ}$ with respect to the $\mathrm{GaN}$ (1120) lattice place, as illustrated in Fig. 1(a). The distance from the substrate to the complementary metal oxide semiconductor (CMOS) detector is set to $523 \mathrm{~mm}$. A high-resolution CMOS detector with an effective pixel size of $5.3 \times 5.3 \mu \mathrm{m}^{2}$ is employed with a resolution of $1920 \times 1440$ pixels. By comparing the observed Laue spots with the calculations, we determine the orientation of the $\mathrm{GaN}$ with respect to the incident $\mathrm{x}$-ray beam. The calculated diffracted $\mathrm{x}$-ray wavelength for the GaN $11 \overline{2} 0$ Bragg condition is $0.1595 \AA$. After determining the Miller indices and diffracted $\mathrm{x}$-ray energy, we adjust the beam size to $8 \mu \mathrm{m}$ (vertical) $\times$ $11 \mathrm{~mm}$ (horizontal) for the section transmission geometry. Here, we assume that the lattice is a nearly perfect crystal but tilted with almost small $d$-spacing variations. By moving the sample downward along the $z$ direction, we obtain the line-shaped image in a plane that is made with $x$ of a sample horizontal position and $y$ of a diffracted wavelength, as shown in Fig. 1(b).

It is not easy to decompose the lattice-tilting vector components into two along the $x$ and $z$ directions unlike the micro x-ray diffraction technique. In the case of our sample with a small tilting angle, the peak positions mainly change to the $y$ direction along the wavelength direction instead of the $x$ direction, as shown in Fig. 1(b). This result means that the diffracted $\mathrm{x}$-ray beam direction change is more sensitive to the tilting along the $x$ axis than the $z$ axis. At arbitrary $z$ points, the lines fluctuated because of the lattice-plane tilt. If the origin of this peak shift were a change of the lattice constant, the variance in the detector would be much smaller than the data we obtain. Because we employ white $\mathrm{x}$ rays as the light source, the change
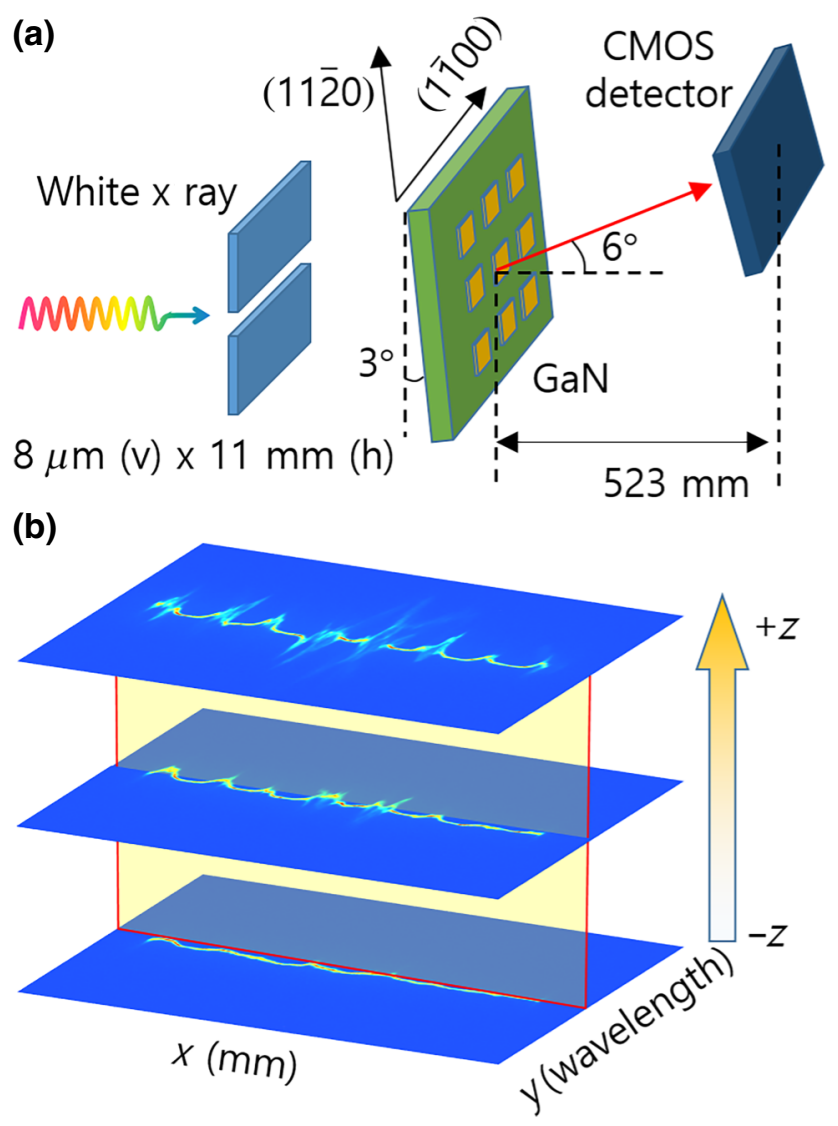

FIG. 1. (a) Schematic illustration of experimental setup. The $\mathrm{GaN}$ surface is irradiated with white $\mathrm{x}$ rays with transmission Laue geometry at an incident angle of $3^{\circ}$. The incident beam size is set to $8 \mu \mathrm{m} \times 11 \mathrm{~mm}$. (b) Images obtained from this configuration are observed with fluctuation because of the local tilting of the lattice plane. By sectioning the image along the $z$ direction (red box), we can obtain a lattice plane image with the same energy.

of the peak position mainly originates from the diffracted energy change, which we can interpret as originating from the lattice-plane tilting instead of a $d$-spacing change. We assume that the sample has a single-crystalline domain along its depth direction. Under the tilting condition, we can rewrite Bragg's law as follows:

$$
2 d \sin \left(\theta_{B}+\theta_{T}\right)=\lambda
$$

This formula becomes the normal Bragg's law if $\theta_{T}$ is equal to 0 . Because of the $\mathrm{x}$-ray energy change by the tilting angle $\theta_{T}$, we can derive the relation between the diffracted $\mathrm{x}$-ray energies and tilting angle. This phenomenon can be explained by introducing the following derivative of Bragg's law:

$$
2 d \cos \left(\theta_{B}+\theta_{T}\right) d \theta_{T}=d \lambda .
$$

Here, $\theta_{B}$ and $\theta_{T}$ represent the Bragg angle and tilting angle, respectively. In this condition, we can evaluate the relative 
diffracted wavelength change related to the peak shift. If we assume that the peak position change originates from the $d$-spacing change with constant diffracted energy, the value is so large that it cannot be acceptable for the GaN substrate. By stacking the image at each $z$ position, we can slice the image at the same wavelength plane, as a yellowfilled rectangular shown in Fig. 1(b), which contains the same wavelength of diffracted $x$ rays. We can then decompose the broadband image into a monochromatic image using this method.

\section{RESULTS AND DISCUSSION}

Figure 2(a) shows an image stacked at $100-\mu \mathrm{m}$ increments of sample $z$ positions. Here, peak position fluctuations are mainly originated from the diffracted energy variation. The evaluated energy change along the yellow dotted line in Fig. 2(a) is shown in Fig. 2(b). The corresponding wavelength changes at $\Delta z=-100 \mu \mathrm{m}$ and 0 are about $-3 \times 10^{-3}$ and $2 \times 10^{-3} \AA$, respectively. In the case of the higher crystalline area shown in Fig. 2(c), the diffracted x-ray energy does not change much. If we consider the main contribution of the peak position changes to the changes of $d$ spacings, the amount of lattice constant change $0.045 \AA$ cannot be acceptable due to the large discrepancy. Moreover, this discrepancy become severe as the $2 \theta$ increases. For this reason, we can convert the peak position change to the change of diffracted x-ray energy originated from lattice tiltings.

Figure 3(a) presents an image of the diffracted $\mathrm{x}$ rays at $\delta \lambda=+0.0016 \AA$. The peak position in the CMOS detector is approximately 100 pixels away from the GaN (1120) Bragg's peak at $3^{\circ}$, which corresponds to a $\theta_{T}$ value of $+0.029^{\circ}$. The periodic structure originates from the fabrication process to release the stress in the GaN substrate. The bottomless structure is seen in the figure. Even though the structure has a symmetrical shape, the bottom of the square parts does not satisfy the Bragg condition. This effect is reversed for the higher energy $\mathrm{x}$ ray of $\delta \lambda=$ $-0.0016 \AA$, whose corresponding $\theta_{T}$ is equal to $-0.029^{\circ}$, as shown in Fig. 3(b). The gradual change of diffracted images from lower x-ray energy to higher $\mathrm{x}$-ray energy can be seen in Video 1 (see also Supplemental Material [24]). The structure has a topless feature, which indicates that the bottom side does not satisfy the Bragg condition at this energy. These bottomless and topless features are also observed when we rotate the sample $90^{\circ}$. The diffracted crystal plane of this condition is GaN (22̄00). Reconstructed images of diffracted $x$ rays under this condition are shown in Figs. 3(c) and 3(d), which show similar features compared to those of $\mathrm{GaN}(1120)$. We could interpret this result as the topless features originating from the diffracted $x$ rays of longer wavelength shown in Fig. 3(e). For short wavelengths, the overall feature is in a lower position in the detector than at longer wavelengths, as
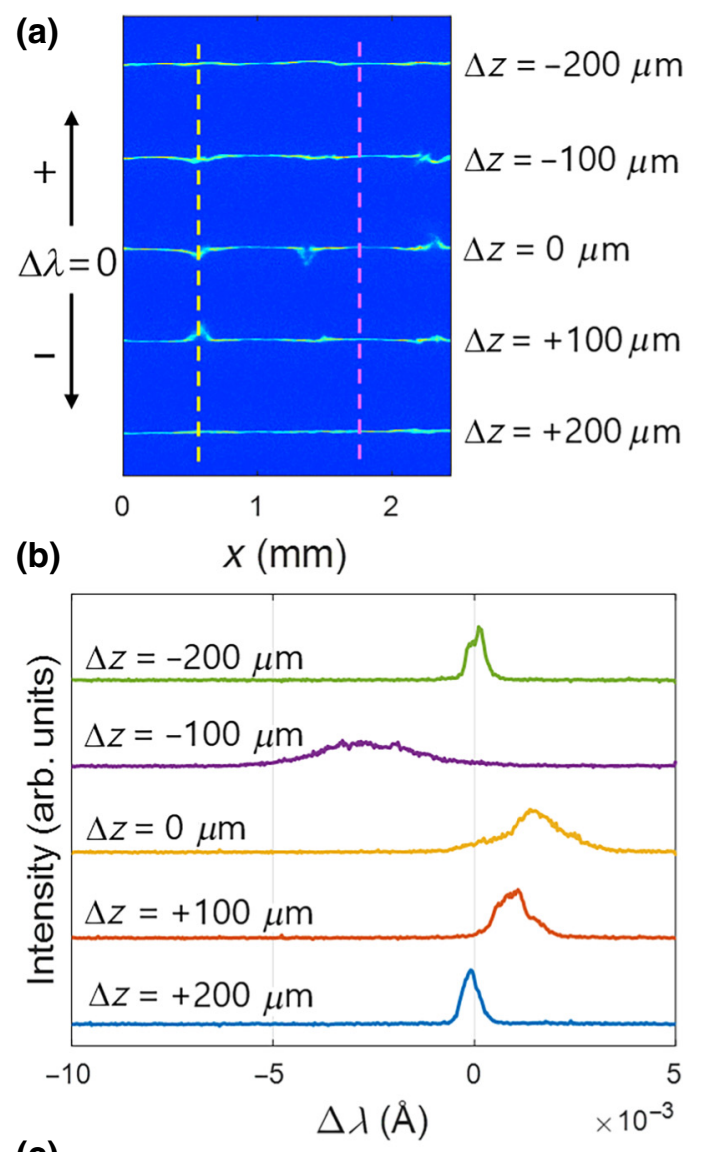

(c)

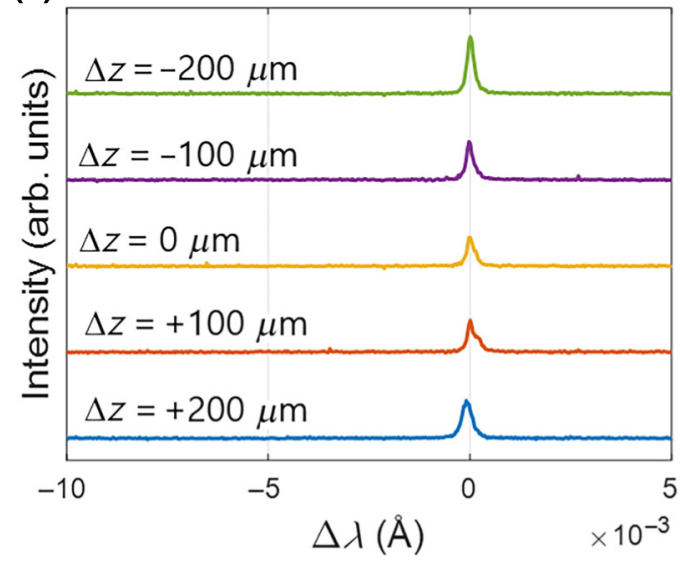

FIG. 2. (a) Diffracted signals at different $z$ positions. Line profiles of the lower crystalline area along the yellow dotted line and the relatively higher crystalline area along the red-dotted line are described in (b) and (c), respectively. The $\Delta z$ values are converted into a corresponding wavelength change.

illustrated in the left side of Fig. 3(e). Therefore, the overall feature should have the shape of symmetrical tilting with the square schematically shown in the right side of Fig. 3(e).

The periodic square shapes are deeply related to the $\mathrm{GaN}$ growth technique. For freestanding $\mathrm{GaN}$, seed crystals are grown on the foreign materials such as $\mathrm{NdGaO}_{3}$ [25]. 
(a)
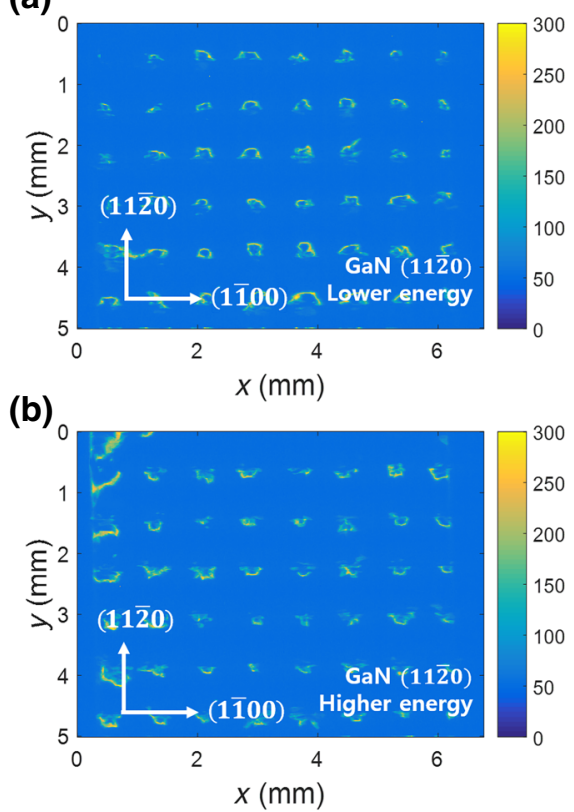

(c)

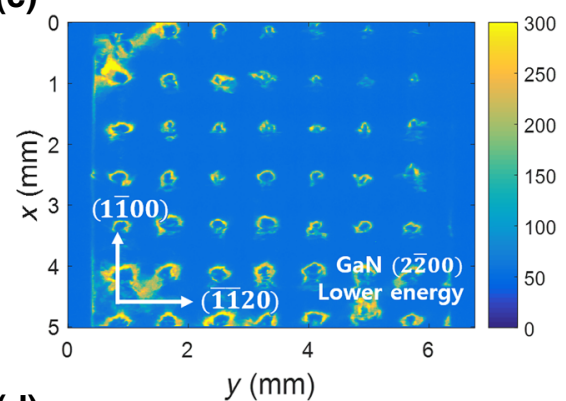

(d)

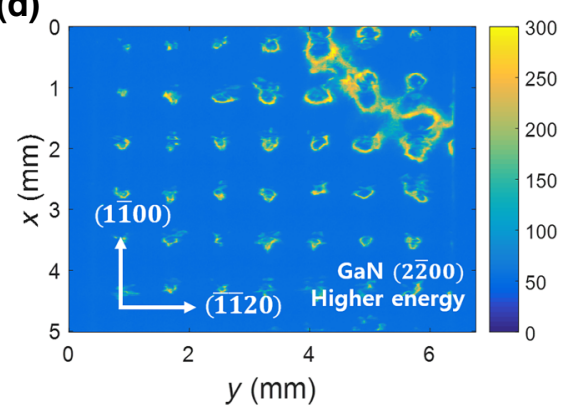

(e)

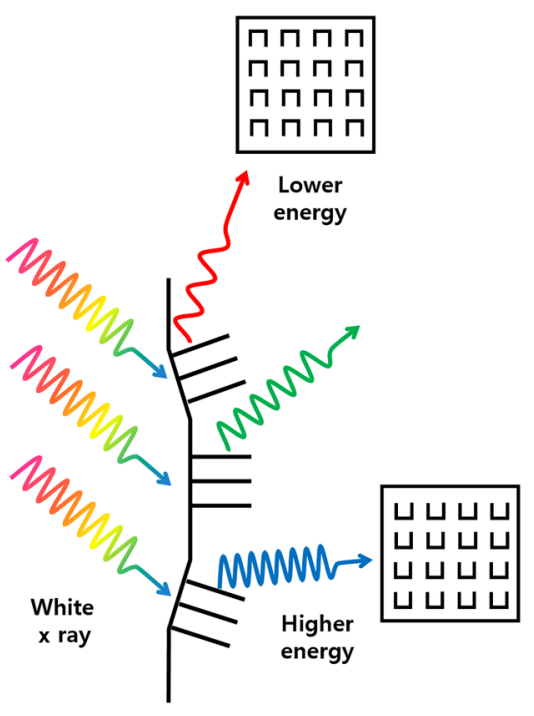

FIG. 3. Reconstructed image at the lower diffraction energy (a),(c) and the higher diffraction energy (b),(d) diffracted from GaN $(11 \overline{2} 0)$ and $\mathrm{GaN}$ (2) 200$)$. The bottomless and topless features originate from the lattice tilting, which generates diffraction $\mathrm{x}$ rays of different energies, as illustrated in (e). The overall shape of the GaN substrate is shown in (e).

In this condition, the HVPE technique can be employed to grow the seed crystals. As the seed crystal grows, they grow laterally to form epitaxial laterally overgrown (ELO) GaN. It is reported that there exist lattice mismatches in the boundary region that split the x-ray rocking curve peak [26]. According to Bertram et al. [27], the ELO area has a larger strain and it is related with impurities. Understanding the correlation between strain relaxation and impurity is important to control and manufacture high-quality and homogeneous freestanding $\mathrm{GaN}$ wafers.

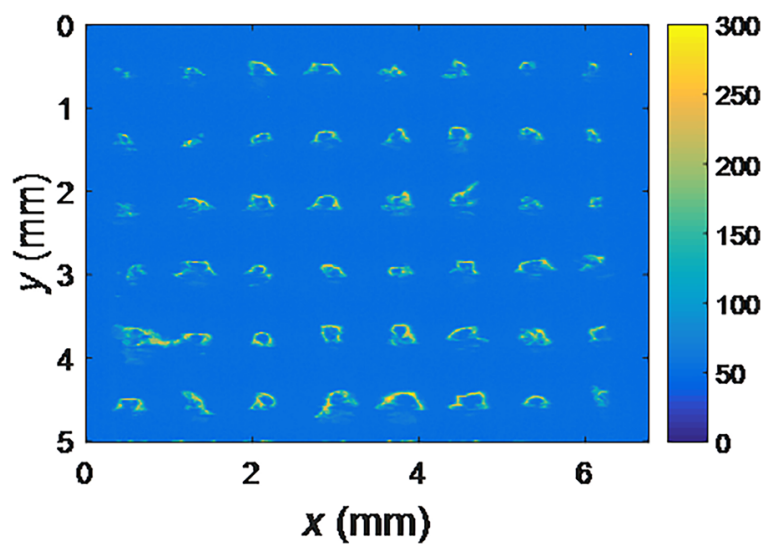

VIDEO 1. Sequential view of reconstructed images from lower energy to higher energy. Periodic structure originated from lattice tilting shows larger intensity.
The lattice-plane orientation tilting is confirmed by monochromatic x-ray diffraction topography performed at BL20B2, SPring-8, Japan [24]. Here, the incident angle is set to $0.58^{\circ}$ and $2 \theta$ is set to $79.3^{\circ}$, which satisfies the (112 4$)$ Bragg condition. We are able to obtain a rocking curve map [28] using a recently developed image-stacking algorithm, as illustrated in Fig. 4(a). The scale bar indicates the lattice tilting angle determined by rocking curve imaging of the GaN (1124) lattice plane. The blue and red dotted patterns originate from the local tilting of the lattice during the substrate fabrication. To determine the tilting angle, we calculate the angle difference between the red and blue parts in Fig. 4(a). The estimated value is approximately $\pm 0.03^{\circ}$, which is consistent with the results obtained from the energy-resolved Laue diffraction topography analysis. We also evaluated the full width at half maximum (FWHM) to estimate the crystal quality shown in Fig. 4(b). The map shows the relatively low crystallinity in the tilted area of the lattice and high crystallinity in the untilted area of the lattice. The average value in the tilted area is $0.07^{\circ}$, which is slightly larger than that in the other region. These results are obtained for the $\pm 0.03^{\circ}$ tilting of the lattice, which enlarges the FWHM.

We examine the crystal quality of the same GaN sample using single crystal $x$-ray diffraction profiles. The experiment is performed at the NIMS BL15XU at SPring-8 using an incident wavelength of $1 \AA$. Monochromatic $\mathrm{x}$ rays are selected using a $\mathrm{Si}(111)$ double-crystal monochromator. The harmonics are completely removed using a mirror system. A six-circle $\mathrm{x}$-ray diffractometer is employed for 

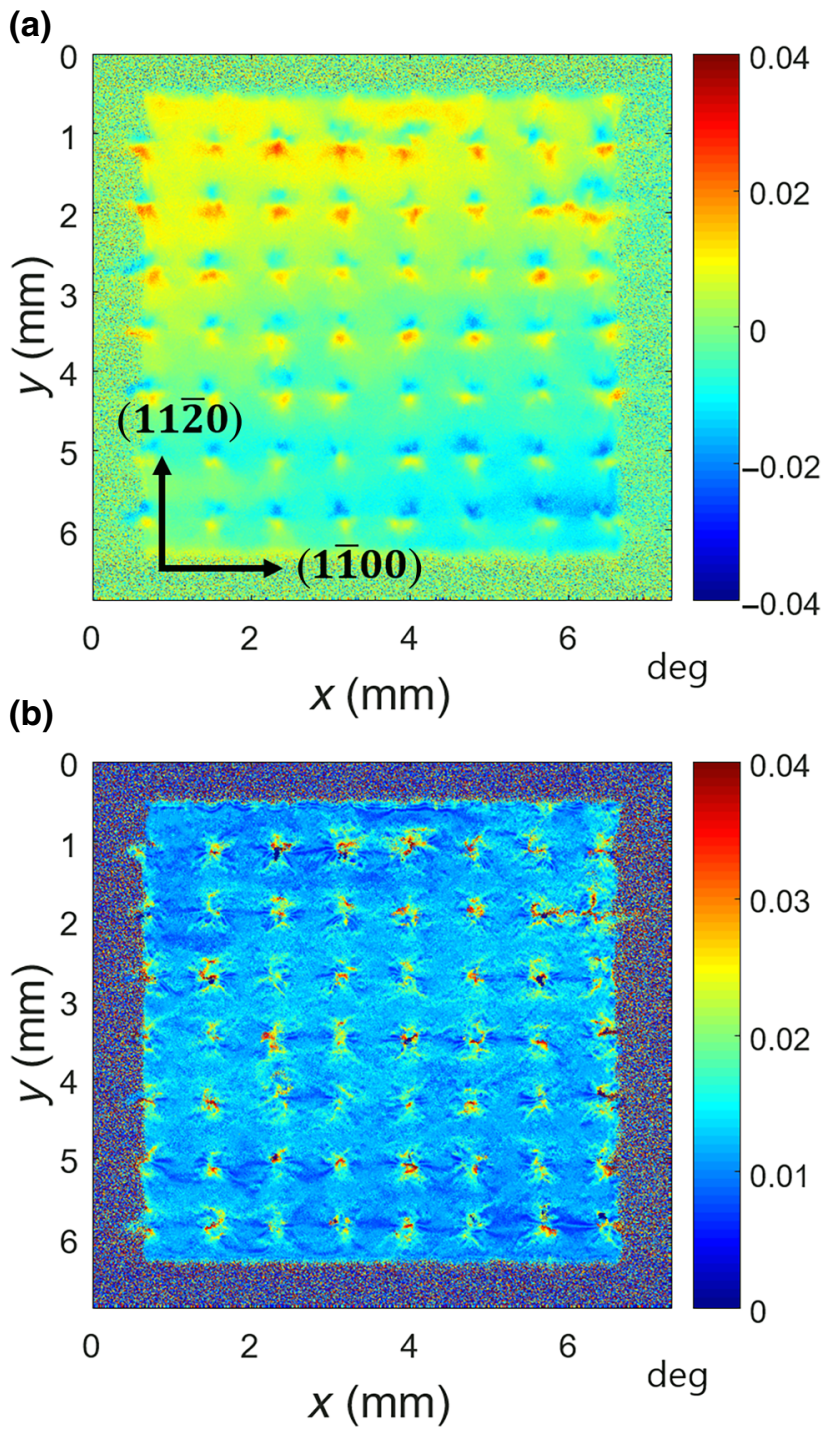

FIG. 4. Monochromatic x-ray diffraction topography image. (a) Image obtained from the evaluation of the peak position of $\theta$ at every point in the sample for the GaN (1124) lattice plane. (b) FWHM map of the GaN substrate. The regular feature is attributed to the mosaicity originating from the substrate fabrication.

the diffraction measurement. The beam size is adjusted to be smaller than $200 \mu \mathrm{m}$ in the horizontal and vertical directions to fully cover one periodic structure of the $\mathrm{GaN}$ substrate along the $y$ direction. The GaN (0002) Bragg angle at this wavelength is $22.25^{\circ}$ with an illumination length on the sample surface of approximately 1 $\mathrm{mm}$ [24]. This length covers approximately two periods of the square structure appearing on the GaN substrate along the beam direction. Under this condition, by translating the $\mathrm{GaN}$ substrate perpendicular to the beam direction, we obtain a high-resolution rocking curve profile as well as a $\theta-2 \theta$ scan $\left(q_{z}\right.$ scan $)$ at some specific points of the substrate. Figure 5 (a) shows the $\theta-2 \theta$ scan of the $\mathrm{GaN}$
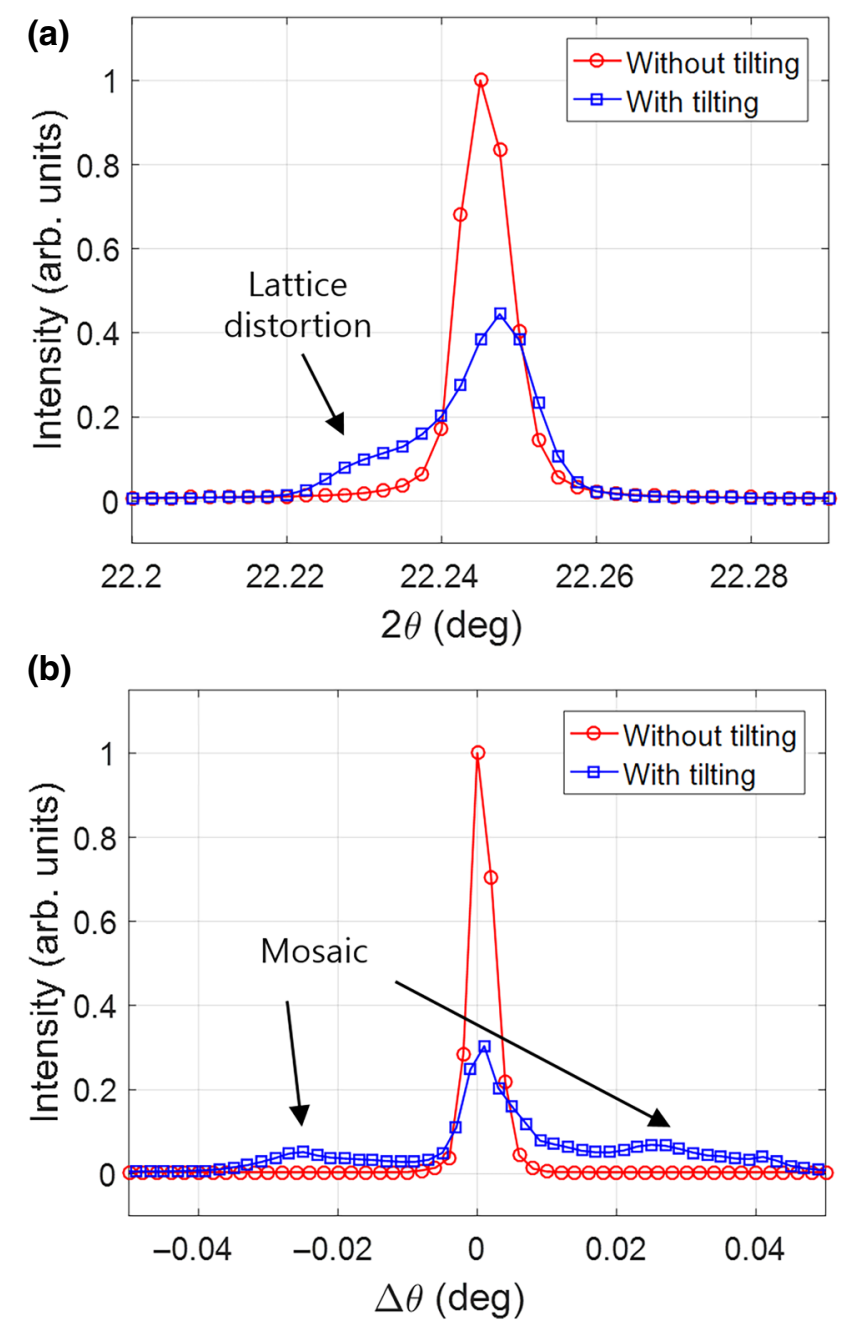

FIG. 5. X-ray diffraction signals of untilted (red) and tilted (blue) areas. (a) $\theta-2 \theta$ scan results. One sharp peak is observed for the untilted parts. In the tilted region, the peak is split into two peaks. (b) $\theta$-rocking curves in the tilted region. In the untilted region, one peak with a FWHM of $0.004^{\circ}$ (red) is observed. However, a mosaic structure with $\pm 0.03^{\circ}$ is observed (blue).

substrate of the untilted (red) and tilted (blue) area. For the untilted part, the peak position is $22.25^{\circ}$, with a corresponding $d$ spacing of $2.5925 \AA$. However, in the tilted region, the peak is split into two peaks at $22.25^{\circ}$ and $22.24^{\circ}$. Accordingly, the evaluated $d$ spacings are 2.5925 and $2.5913 \AA$, respectively. The peak position difference is approximately $0.01^{\circ}$, which is undistinguishable using single-crystal $\mathrm{x}$-ray diffraction with rotating anode $\mathrm{x}$ rays because of the resolution limitation. The evaluated $\delta d / d$ is approximately $4.6 \times 10^{-4}$, which is small enough that we can consider this GaN substrate to be a high-quality single crystal even though the edge of the patterned parts is slightly tilted, as shown in Fig. 5(b). As expected from the previous results, some distribution is observed in the square-shaped region because of the lattice tilting. The tilting angle is estimated to be $\pm 0.03^{\circ}$, which is consistent 
with the results obtained using the energy-resolved Laue $\mathrm{x}$-ray diffraction topography method, which uses white $\mathrm{x}$ rays. The satellite peaks originate from the lattice tilting, but they are not observed in the untilted region. For the untilted region, the FWHM is approximately $0.004^{\circ}$, which is similar to the findings in a previous report [8].

Using x-ray diffraction topography and the photoluminescence of $\mathrm{GaN}$, the number of dislocations can be evaluated using image analysis [8]. However, even though the overall feature can be observed, the lattice tilting and distribution on a wafer scale cannot be determined. In our experiment, we show the lattice tilting, which is related to the lattice tilting by the rocking curve map. By magnifying the image through a lens and using a phosphor CCD detector, we expect to be able to achieve high image resolution; however, we may not see the overall feature on the wafer scale. The misorientation deformation (mosaicity) and dislocations in $\mathrm{GaN}$ are shown using synchrotron white-beam x-ray topography (SWXRT) on a GaN substrate using back reflection Laue geometry [29]. The report does not show the lattice tilting on a wafer scale even though they adopted section transmission geometry, which means that we improve the SWXRT technique for the observation of tilting on the wafer scale in the current work. In the case of monochromatic x-ray topography, photon flux is much smaller than white $\mathrm{x}$ rays due to its narrow bandwidth. The photon flux becomes worse if we employ transmission geometry for bulk sensitive information. Our proposed method directly uses white or polychromatic $\mathrm{x}$ rays without wavelength filtering, which reduces the wafer inspection time. Although our proposed method is sensitive to the lattice-plane tilting suitable for high-quality crystalline material, it is not so sensitive to the $d$-spacing variation. Therefore, new techniques that fully cover lattice-plane tiltings and $d$-spacing variations are in demand to understand the crystallinity of wafers more precisely.

\section{CONCLUSION}

In summary, we examine lattice-plane orientation tilting using an energy-resolved white and Laue x-ray diffraction topography technique. The tilting angle of a GaN sample is determined to be $\pm 0.03^{\circ}$, which is consistent with the $\mathrm{x}$-ray diffraction topography results. Good agreement is observed between the energy-resolved Laue x-ray images and both single-crystal x-ray diffraction results and monochromatic $\mathrm{x}$-ray diffraction topography images. We expect that this technique will be useful for estimation of crystal quality on the wafer scale.

\section{ACKNOWLEDGMENTS}

The measurements are performed at SPring- 8 under Grants No. 2016B1028, No. 2017A1030, No. 2017B1029, No. 2017B4505, and No. 2017B1030. This work is supported by the Ministry of Education, Culture, Sports, Science and Technology (MEXT) "Program for Research and Development of Next-Generation Semiconductor to Realize Energy-Saving Society."

[1] H. P. Maruska and J. J. Tietjen, The preparation and properties of vapor deposited single crystaline GaN, Appl. Phys. Lett. 15, 327 (1969).

[2] M. A. Khan, A. Bhattarai, J. N. Kuznia, and D. T. Olson, High electron mobility transistor based on a GaN-Al $\mathrm{Ga}_{1-x} \mathrm{~N}$ heterojunction, Appl. Phys. Lett. 63, 1214 (1993).

[3] S. Nakamura, T. Mukai, M. Senoh, and N. Iwasa, Thermal annealing effects on P-type Mg-doped GaN films, Jpn. J. Appl. Phys. 31, L139 (1992).

[4] K. Motoki, T. Okahisa, S. Nakahata, N. Matsumoto, H. Kimura, H. Kasai, K. Takemoto, K. Uematsu, M. Ueno, Y. Kumagai, A. Koukitu, and H. Seki, Growth and characterization of freestanding GaN substrates, J. Cryst. Growth 237-239, 912 (2002).

[5] R. Dwilinśki, R. Doradzinśki, J. Garczynśki, L. P. Sierzputowski, A. Puchalski, Y. Kanbara, K. Yagi, H. Minakuchi, and H. Hayashi, Excellent crystallinity of truly bulk ammonothermal GaN, J. Cryst. Growth 310, 3911 (2008).

[6] R. Dwilinśki, R. Doradzinśki, J. Garczynśki, L. P. Sierzputowski, A. Puchalski, Y. Kanbara, K. Yagi, H. Minakuchi, and H. Hayashi, Bulk ammonothermal GaN, J. Cryst. Growth 311, 3015 (2009).

[7] Y. Cui, X. Hu, X. Xie, R. Wang, and X. Xu, Spatial variation of lattice plane bending of $4 \mathrm{H}-\mathrm{SiC}$ substrates, CrystEngComm 19, 3844 (2017).

[8] N. A. Mahadik, S. B. Qadri, and J. A. Freitas Jr., Structural inhomogeneities and impurity incorporation in growth of high-quality ammonothermal GaN substrates, Cryst. Growth Des. 15, 291 (2015).

[9] S. Kikuta, K. Kohra, and Y. Sugita, Measurements on local variations in spacing and orientation of the lattice plane of silicon single crystals by X-ray double-crystal topography, Jpn. J. Appl. Phys. 5, 1047 (1966).

[10] T. Ishikawa, T. Kitano, and J. Matui, Equi-lattice-spacing mapping x-ray topography, J. Appl. Cryst. 20, 344 (1987).

[11] C. Ferrari, D. Korytar, and J. Kumar, Study of residual strains in wafer crystals by means of lattice tilt mapping, Il Nuovo Cimento D 19, 165 (1997).

[12] A. T. Macrander, S. Krasnicki, Y. Zhong, J. Maj, and Y. S. Chu, Strain mapping with parts-per-million resolution in synthetic type-Ib diamond plates, Appl. Phys. Lett. 87, 194113 (2005).

[13] J. Guo, Y. Yang, B. Raghothamachar, M. Dudley, and S. Stoupin, Mapping of lattice strain in $4 \mathrm{H}-\mathrm{SiC}$ crystals by synchrotron double-crystal x-ray topography, J. Electron. Mater. 47, 903 (2017).

[14] J. Kim, O. Seo, C. Song, S. Hiroi, Y. Chen, Y. Irokawa, T. Nabatame, Y. Koide, and O. Sakata, Lattice-plane orientation mapping of homo-epitaxial $\mathrm{GaN}(0001)$ thin films via grazing-incidence $x$-ray diffraction topography in 2-in. wafer, Appl. Phys. Express 11, 081002 (2018). 
[15] J. Kim, O. Seo, C. Song, Y. Chen, S. Hiroi, Y. Irokawa, T. Nabatame, Y. Koide, and O. Sakata, Characterization of a 4-inch GaN wafer by x-ray diffraction topography, CrystEngComm 20, 7761 (2018).

[16] B. C. Larson, W. Yang, G. E. Ice, J. D. Budai, and J. Z. Tischler, Three-dimensional x-ray structural microscopy with submicrometre resolution, Nature 415, 887 (2002).

[17] W. Liu, G. E. Ice, B. C. Larson, W. Yang, J. Z. Tischler, and J. D. Budai, The three-dimensional x-ray crystal microscope: A new tool for materials characterization, Metall. Mater. Trans. A 35A, 1964 (2004).

[18] G. E. Ice and B. C. Larson, 3D X-ray crystal microscope, Adv. Eng. Mater. 2, 643 (2000).

[19] L. Margulies, G. Winther, and H. F. Poulsen, In situ measurement of grain rotation during deformation of polycrystals, Science 291, 2392 (2001).

[20] R. Barabash, G. E. Ice, B. C. Larson, G. M. Pharr, K. S. Chung, and W. Yang, White microbeam diffraction from distorted crystals, Appl. Phys. Lett. 79, 749 (2001).

[21] W. M. Chena, P. J. McNallya, K. Jacobsb, T. Tuomic, A. N. Danilewskyd, Z. R. Zytkiewicze, D. Lowneya, J. Kanatharanaa, L. Knuuttilac, and J. Riikonen, Determination of crystal misorientation in epitaxial lateral overgrowth of GaN, J. Cryst. Growth 243, 94 (2002).

[22] P. Tafforeau, R. boistel, E. Boller, A. Bravin, M. Brunet, Y. Chaimanee, P. Cloetens, M. Feist, J. Hoszowska, J.-J. Jaeger, R. F. Kay, V. Lazzari, L. Marivaux, A. Nel, C. Nemoz, X. Thibault, P. Vignaud, and S. Zabler, Applications of x-ray synchrotron microtomography for nondestructive 3D studies of paleontological specimens, Appl. Phys. A 83, 195 (2006).

[23] X. R. Huang, LauePt, a graphical-user-interface program for simulating and analyzing white-beam x-ray diffraction Laue patterns, J. Appl. Cryst 43, 926 (2010).
[24] See Supplemental Material at http://link.aps.org/supple mental/10.1103/PhysRevApplied.11.024072 for periodic structures originated from lattice tilting that show larger intensity, the experimental configuration of monochromatic x-ray diffraction topography (Fig. S1), images obtained at various angles (Fig. S2), Bragg (reflection) geometry for choosing small areas of untilted and tilted regions on a $\mathrm{GaN}$ wafer (Fig. S3).

[25] A. Wakahara, T. Yamamoto, K. Ishio, A. Yoshida, Y. Seki, K. Kainosho, and O. Oda, Hydride vapor phase epitaxy of $\mathrm{GaN}$ on $\mathrm{NdGaO}_{3}$ substrate and realization of freestanding GaN wafers with 2-inch scale, Jpn. J. Appl. Phys. 39, 2399 (2000).

[26] Z. R. Zytkiewcz, Laterally overgrown structures as substrates for lattice mismatched epitaxy, Thin Solid Films 412, 64 (2002).

[27] F. Bertram, T. Riemann, J. Christen, A. Kaschner, A. Hoffmann, C. Thomsen, K. Hiramatsu, T. Shibata, and N. Sawaki, Strain relaxation and strong impurity incorporation in epitaxial laterally overgrown GaN: Direct imaging of different growth domains by cathodoluminescence microscopy and micro-Raman spectroscopy, Appl. Phys. Lett. 74(3), 359 (1999).

[28] D. Lübbert, C. Ferrari, P. Mikulík, P. Pernot, L. Helfen, N. Verdi, D. Korytár, and T. Baumbach, Distribution and Burgers vectors of dislocations in semiconductor wafers investigated by rocking curve imaging, J. Appl. Cryst. 38, 91 (2005).

[29] L. Kirste, A. N. Danilewsky, T. Sochacki, K. Köhler, M. Zajac, R. Kucharski, M. Boćkowski, and P. J. McNally, Synchrotron white-beam X-ray topography analysis of the defect structure of HVPE-GaN substrates, ECS J. Solid State Sci. Technol. 4(8), P324 (2015). 